\title{
Current Status and Future Directions in the Management of Pancreatic Cancer
}

\author{
Chandrakanth Are • Asish Patel
}

Received: 8 December 2014 / Accepted: 12 January 2015 / Published online: 25 January 2015

(C) Indian Association of Surgical Oncology 2015

Worldwide there were 337,872 new cases of pancreatic cancer diagnosed in 2012 with 330,372 deaths and a prevalence rate of 211,544 [1,2] (Table 1). The incidence, mortality and prevalence rates are higher in males when compared to females worldwide. The number of newly diagnosed cases varies very widely between countries of low versus high incidence. The incidence of pancreatic cancer is low in India when compared to the United States (U.S). Except for the incidence rate in females, India falls into the lowest quintile in terms of incidence (males), mortality (males and females) and prevalence (males and females). In contrast, the United States falls into the top most quintile for incidence, mortality and prevalence of pancreatic cancer in both sexes $[1,2]$.

Pancreatic cancer is the fourth leading cause of cancerrelated deaths in both males and females in the United States, only to be surpassed by cancers involving the lung/bronchus, breast, prostate, and colon/rectum [3]. Pancreatic cancer accounts for nearly $7 \%$ of all cancer-related deaths in the United States [3]. Pancreatic cancer also stands 9th in the number of estimated new cancer cases diagnosed in females in 2014 , accounting for $3 \%$ of all new malignancies [3]. In contrast, a review of the 12 population based cancer registries (PBCRs) from India (Benaguluru, Barshi, Bhopal, Chennai, Delhi, Mumbai, Ahmedabad, Karunagappally, Kolkata, Nagpur, Pune and Thiruvananthapuram) demonstrates that the incidence of pancreatic cancer is significantly lower than in the U.S $[4,5]$. Although the incidence in India is presently lower than that of the U.S, the rate of rise in new cases of pancreatic cancer in the future will be much higher in India [6]. It is

C. Are $(\triangle) \cdot$ A. Patel

Fred and Pamela Buffett Cancer Center, Division of Surgical

Oncology, Department of Surgery, University of Nebraska Medical

Center, 4400 Emile St, Omaha, NE 68198, USA

e-mail: care@unmc.edu estimated that by 2035 the number of new cases will increase by nearly $89 \%$ in India when compared to $55 \%$ in the US [6].

Although surgical resection offers the only hope of prolonging survival, few patients (15-20\%) present at a stage that is suitable for operative intervention. Of the patients that present with resectable disease, not everyone undergoes operative resection, either due to poor performance status or failure to access care at a tertiary care center with the required expertise. The mortality rates associated with pancreatic surgery have improved significantly since the early 1970's due to a combination of various factors such as improved surgical techniques, better anesthesia and critical care management, concentration of care at tertiary care centers, and improved ability to deal with life-threatening complications. Although the mortality rates have improved, the morbidity associated with pancreatic resections is still considerable up to $50 \%$. The article by Penumadu et al. provides a review of how to prevent and treat adverse outcomes related to haemorrhage, pancreatic fistula, delayed gastric emptying and bile leaks. The improved mortality rates and ability to address adverse events has prompted some groups to advocate for more aggressive resections that go beyond that standard pancreaticoduodenectomy. The article by Schoellhammer et al. discusses the issues related to extended lymphadenectomy and vascular resections with reconstructions.

Several advances have been made over the past few decades in the management of pancreatic cancer. Although most of these have been relevant to the actual treatment, we have also seen advances in imaging and definitions and staging. The historical bi-categories of resectable and unresectable have been further subdivided into resectable, borderline resectable, locally advanced, and unresectable. The article by Mullapudi and Hawkes et al. discusses the definitions and management of the newer entity called borderline resectable pancreatic cancer. The other advances made in pancreatic surgery include the introduction of minimally invasive surgical 
Table 1 Incidence, mortality, and prevalence of pancreatic cancer [1-6]

\begin{tabular}{|c|c|c|c|c|}
\hline & & Worldwide & India & US \\
\hline \multirow[t]{3}{*}{ Incidence (number of cases) } & Male & 178,161 & 6,582 & 21,713 \\
\hline & Female & 159,711 & 5,354 & 21,712 \\
\hline & Total & 337,872 & 11,936 & 43,425 \\
\hline \multirow[t]{2}{*}{ Incidence (age- standardized incidence rate/100,000) } & Male & 4.9 & 1.3 & 8.6 \\
\hline & Female & 3.6 & 1 & 6.5 \\
\hline \multirow[t]{3}{*}{ Mortality (number of cases) } & Male & 173,812 & 5,934 & 21,005 \\
\hline & Female & 156,560 & 4,894 & 20,504 \\
\hline & Total & 330,372 & 10,828 & 41,509 \\
\hline \multirow[t]{2}{*}{ Mortality (age-standardized mortality rate/100,000) } & Male & 4.8 & 1.2 & 8.2 \\
\hline & Female & 3.4 & 0.9 & 5.9 \\
\hline \multirow[t]{3}{*}{ Prevalence (number of cases alive with diagnosis of cancer within previous 5 year) } & Male & 114,434 & 3,710 & 12,984 \\
\hline & Female & 97,110 & 3,020 & 12,027 \\
\hline & Total & 211,544 & 6,730 & 25,011 \\
\hline \multirow[t]{2}{*}{ Prevalence (age-standardized proportion/100,000) } & Male & 4.4 & 0.8 & 10.5 \\
\hline & Female & 3.7 & 0.7 & 9.3 \\
\hline
\end{tabular}

techniques with laparoscopy and robotic surgery. Pancreatic surgery remained the last bastion to the advances of minimally invasive surgery. An increasing number of centers across the world are performing laparoscopic/robotic pancreatic resections and have reported comparable outcomes in highly selected patients. The article by Senthilinathan et al. provides a comparison between open and laparoscopic pancreatic resections and document that in centers with available expertise the outcomes are similar.

We have come a long way from the era of 5 -fluorouracil which since its discovery in the 1950's had remained the mainstay for chemotherapy for gastrointestinal cancers, including pancreatic cancer till the introduction of gemcitabine. The role of chemotherapy has changed in numerous aspects such as: a) timing of administration with increasing emphasis on pre-operative administration, b) different types of combination regimes such as FOLFIRINOX and c) different combination approaches with radiation therapy. Although studies in the U.S have shown the benefit of combining radiation with chemotherapy, this has not been duplicated in European studies. The article by Sirohi et al. provides a review of the current drugs and combinations available to treat patients with pancreatic cancer in all settings.

Lastly, the diagnosis of pancreatic cancer can still be problematic even in the era of triple phase imaging and endoscopic capabilities. This can become even more difficult in patients with chronic pancreatitis or in patients from areas where tuberculosis is endemic. The article by Dhar et al. outlines the approach to treat patients with a history of chronic pancreatitis and likely diagnosis of pancreatic cancer.

We have witnessed several advances in the management of pancreatic cancer with improved short-term outcomes. While the short-term outcomes have improved, the long-term outcomes continue to remain dismal except for a select group of patients that receive their care at centers of excellence. We may be close to the peak of surgical excellence as witnessed by the remarkably low mortality rates following resections. Further advances may arrive with the increasing use of minimally invasive and robotic techniques. What is more pressing and needed to swing the pendulum of survival favorably is a better understanding of the biology of the disease. This can help in identifying risk factors, target high risk individual for early diagnosis and define therapeutic targets for developing newer drugs. This is paramount so that it can equip physicians with the tools required to tackle this lethal disease. Finally, all efforts should be placed on maintaining the quality of life in these patients regardless of the treatment intent or stage of disease. This combination of treatment advances and a focus on quality of life should be the preferred approach for treating patients diagnosed with this lethal malignancy.

Conflict of Interest The author declares that they have no conflict of interest.

\section{References}

1. Bray F, Ren JS, Masuyer E, Ferlay J (2013) Estimates of global cancer prevalence for 27 sites in the adult population in 2008. Int J Cancer 132(5):1133-45. doi: 10.1002/ijc.27711. Epub 2012 Jul 26. Available from: http://globocan.iarc.fr/Default.aspx. Accessed 21 Aug 2014

2. Ferlay J, Soerjomataram I, Ervik M, Dikshit R, Eser S, Mathers C, Rebelo M, Parkin DM, Forman D, Bray, F (2013) GLOBOCAN 2012 v1.0, Cancer Incidence and Mortality Worldwide: IARC CancerBase No. 11 [Internet]. Lyon, France: International Agency for Research on Cancer. Available from: http://globocan.iarc.fr/ia/World/atlas.html . Accessed 8/21/2014 
3. American Cancer Society (2014) Cancer Facts \& Figures 2014. http://www.cancer.org/research/cancerfactsstatistics/cancer factsfigures2014/. Accessed 12 Aug 2014.

4. National Cancer Registry Programme (2010) Development of an atlas of cancer in India. First all India report: 2001-2002. http://www. canceratlasindia.org/chapter3_5.aspx. Accessed 21 Aug 2014

5. National Cancer Registry Programme (2010) Development of an Atlas of cancer in India. First all India report: 2001-2002. http://www.canceratlasindia.org/chapter3_6.aspx. Accessed 21 Aug 2014

6. Ferlay J, Soerjomataram I, Ervik M, Dikshit R, Eser S, Mathers C, Rebelo M, Parkin DM, Forman D, Bray, F (2013) GLOBOCAN 2012 v1.0, Cancer incidence and mortality worldwide: IARC CancerBase No. 11 [Internet]. Lyon, France: International Agency for Research on Cancer. Available from: http://globocan.iarc.fr/Pages/burden_sel.aspx. Accessed 25 Aug 2014. 